\title{
La alfabetización digital como factor de inclusión social. La experiencia de la Red \\ Conecta.
}

\author{
Josep Castro Borrallo \\ pcastro@esplai.org \\ Coordinador Red Conecta. Fundación Esplai
}

\section{La Fundación Esplai y la Red Conecta}

La Fundación Esplai es una entidad sin afán de lucro que tiene como fin fundamental la superación de la exclusión social y que articula sus objetivos en torno a la educación de la infancia y la juventud, el desarrollo y fortalecimiento de las asociaciones y la prevención de las drogodependencias.

En su conjunto, cuenta con más de 700 profesionales y 1.500 voluntarios que participan en sus proyectos, programas y servicios. En 2003, las acciones y programas del conjunto de la Fundación han llegado a mas de 350.000 personas en España.

Ha impulsado el proyecto Red Conecta, cuyo objetivo principal es acercar y formar en las TIC a las personas más desfavorecidas, como medida que ha de contribuir a favorecer su inclusión social. En él se combina fortalecimiento asociativo, nuevas tecnologías, inclusión social y educación en valores.

El proyecto se ha materializado a través de la colaboración de los tres sectores, el social, el público y el privado, siendo este uno de los trazos de identidad que lo diferencia de otras iniciativas. Especialmente remarcable ha sido el apoyo de la International Youth Foundation, (IYF).

La Red Conecta se compone actualmente de 41 centros implantados en 14 comunidades autónomas. Y en su globalidad la Fundación coordina 71 centros de acceso y formación en las TIC en los que participan mas 15.000 personas al año[1].

\section{Orientarse es más que usar una brújula}

El que haya usado una brújula sabe que no es suficiente para orientarse. ¿De qué nos sirve saber si el norte está en una determinada dirección si no sabemos a dónde queremos ir, ni en donde estamos? Hay que saber usar la brújula, pero sobre todo, hay que saber observar el entorno, saber de donde partimos y dónde queremos ir, saber preguntar a los vecinos...

Desde nuestra experiencia, con la alfabetización digital pasa un poco lo mismo. No basta con aprender a manejar el teclado y el ratón, o conocer el manejo del procesador de texto, el correo electrónico o el navegador de internet.

Se necesitan pues otras habilidades sociales, culturales, que nos permitan hacer un uso con sentido de estas herramientas, de manera que contribuyan a mejorar nuestra autonomía, nuestra capacidad de comunicarnos, de trabajar, participar... y pasemos del uso a la "apropiación" de la herramienta. Será entonces cuando habremos aprendido a orientarnos.

Para la alfabetización digital orientada a la inclusión social, nos parece evidente la necesidad de esos aprendizajes no tecnológicos para lograr mejores condiciones de inclusión social. Para las personas con menos recursos culturales, sin hábitos de trabajo académico o con una baja autoestima, aprender a usar un ordenador les ayuda a recuperar la confianza en su capacidad de aprender, y ese cambio 
mental es mucho más importante que su habilidad con el procesador de texto.

Hay que apoyarse en ese cambio de actitud, para hacer aflorar el potencial de la persona en sus capacidades de relación con los demás, de trabajo en equipo, de participar, y de construir su propia identidad. La mejora de estas capacidades permite aprovechar mejor las oportunidades del mercado laboral, accediendo a puestos en los que sin experiencia como usuario de informática, no sería posible.

\section{La Brecha Digital}

Una de las definiciones al uso sobre la Brecha Digital es la que la cita como la distancia que existe entre las personas, colectivos sociales y países que tienen acceso a las Tecnologías de la Información y la Comunicación y quienes no lo tienen.

Las razones de esa brecha son multifactoriales, y van desde la simple carencia de conectividad física en el lugar de residencia, como ocurre en zonas rurales y barrios degradados, hasta las barreras mentales que hace que determinadas personas se autoexcluyan de la sociedad de la información.

Naturalmente, también hay razones económicas (incapacidad de asumir los costes), razones metodológicas (la forma de aprender y los contenidos, no interesan ni se sienten como algo propio), y razones tecnológicas (los dispositivos, aplicaciones y usos no están pensados para personas con discapacidades, lenguajes o necesidades diferentes). Pero en realidad, al final, según Castells "la brecha digital, es una manifestación de la brecha social", que refleja una situación de diferencia de acceso, de desigualdad de oportunidades de participación y de comunicación[2].

El acceso a las TIC pues, es fundamental para acceder a la información y al conocimiento y, en consecuencia, para posibilitar la educación, el desarrollo y la inclusión social. Su uso es una oportunidad para multiplicar nuestra capacidad de relación y comunicación.

Las medidas para la alfabetización digital de esas personas deben tener en cuenta estos factores y estamos convencidos que las organizaciones de la sociedad civil tienen un papel destacado para la superación de la brecha digital.

En los centros de la Red Conecta se facilita el aprendizaje de la informática y el acercamiento a las TIC mediante un entorno de proximidad y sentido para las personas.

\section{Modelo de centro Red Conecta.}

\subsection{LAS PERSONAS DESTINATARIAS}

Los centros están ubicados en barrios y zonas desfavorecidas y se dirigen al conjunto de la población, con prioridad hacia las personas y colectivos con dificultades de acceso y baja formación, con prioridad para jóvenes de 13 a 30 años con déficit culturales, sociales y económicos, mujeres con dificultades de inserción socio-laboral, parados de larga duración, etc.

Las personas participantes en los cursos o actividades formativas que se realizan en los centros ven sus progresos y perciben ese aprendizaje como algo accesible, dentro de sus capacidades, y con muchos menos miedos o reservas que los que sentirían ante una oferta de una academia o un centro similar.

\subsection{LAS ENTIDADES COLABORADORAS}

Esta iniciativa se basa en la colaboración entre asociaciones y fundaciones de carácter social con experiencia y posicionamiento en su territorio y en las alianzas con empresas y administraciones públicas, para unir esfuerzos e implicar a todos los sectores de la sociedad en el compromiso por la superación de la brecha digital. 
Las asociaciones locales tienen el conocimiento del entorno, el contacto con las personas, la credibilidad, la proximidad...

En definitiva la capacidad para llegar a las personas, lo cual garantiza un alto nivel de participación en los centros.

Estas entidades son seleccionadas a través de convocatoria y con ellas se establece un acuerdo de colaboración a tres años para el desarrollo del proyecto, por el que la Fundación Esplai aporta los equipos informáticos, una metodología específica, los materiales didácticos, la formación continuada de las personas dinamizadoras y ofrece un soporte de coordinación, seguimiento y evaluación.

Las entidades por su parte, aportan el local donde ubicar el aula, la persona para las funciones de dinamización y un equipo de apoyo de voluntarios y/o profesionales especializados en aspectos de inclusión social, que ofrecen una atencióndiferenciada a cada participante o grupo.

\subsection{LA DINAMIZACIÓN}

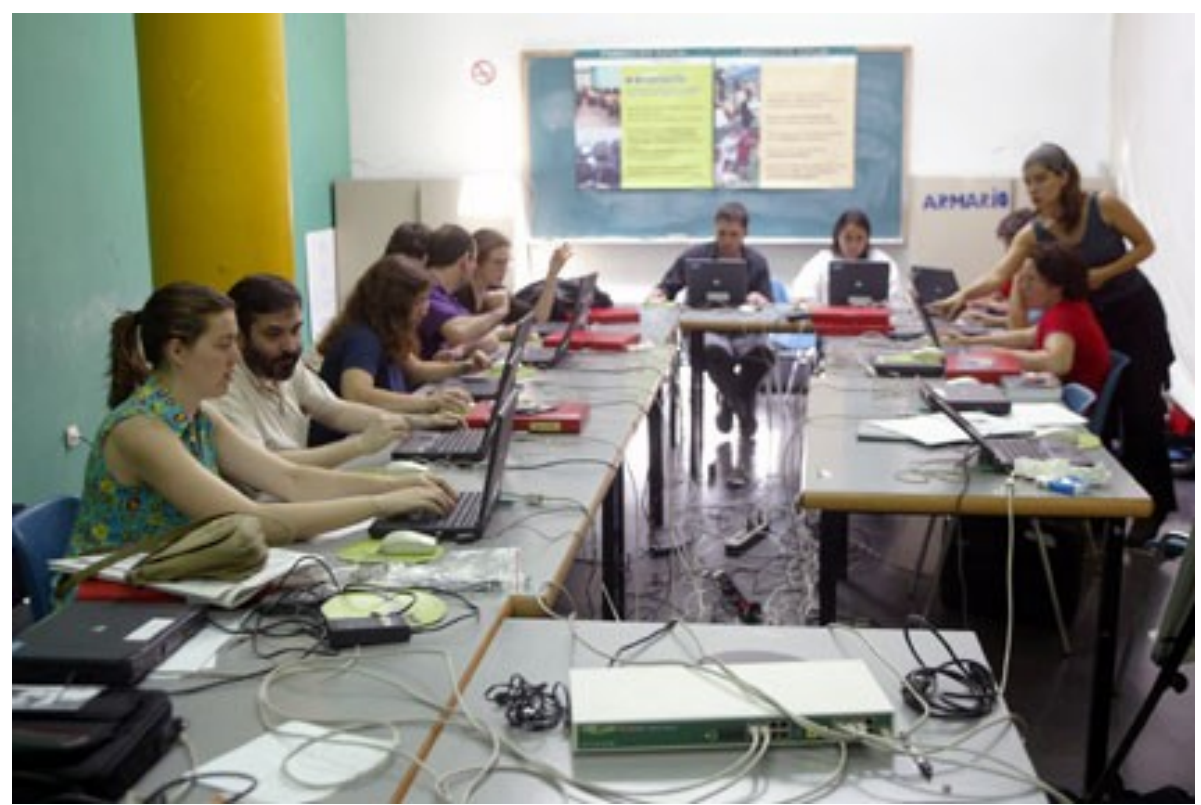

Los dinamizadores, son el elemento clave, pues son quienes establecen el contacto directo con las personas, y su capacidad relacional, de diálogo, motivación y acompañamiento es mucho más relevante que sus conocimientos de informática. Hemos buscado preferentemente educadores, pedagogos, trabajadores sociales, a los que se ha formado en la metodología Red

Conecta y una mínima base tecnológica.

A mayor implicación personal, mejores resultados. Pero hemos de decir que también se precisa reforzar la formación y prestar apoyo metodológico a dinamizadores/as y alfabetizadores/as, dentro y fuera de las organizaciones sociales., por ello, los dinamizadores participan de un proceso de formación y reciclaje permanente.

Las funciones del dinamizador son:

- Potenciar la iniciativa y la creatividad de las personas participantes

- Potenciar la concepción constructivista de los aprendizajes (el mismo usuario es quién va construyendo su conocimiento)

- Crear un ambiente motivador

- Favorecer al aumento de la autoestima y la valoración personal a través de la tutoría individual

- Estimular la interacción grupal potenciando los grupos heterogéneos y el trabajo cooperativo.

- Dar a conocer el centro en las entidades del territorio, invitando y proponiendo actividades

\subsection{EL TRABAJO EN RED}

Existen diversas iniciativas para la superación de la brecha digital, algunas impulsadas por la Administración, otras por parte de las ONG y algunas por parte de las empresas. A menudo, estos proyectos solo buscan puntos y momentos de contacto e intercambio y en algunos casos se plantean en forma de red, pero a la práctica sólo comparten la dotación de equipamiento y la financiación. Desde nuestra experiencia, consideramos que los elementos críticos no son los factores tecnológicos sino los elementos humanos, metodológicos y sociales. 


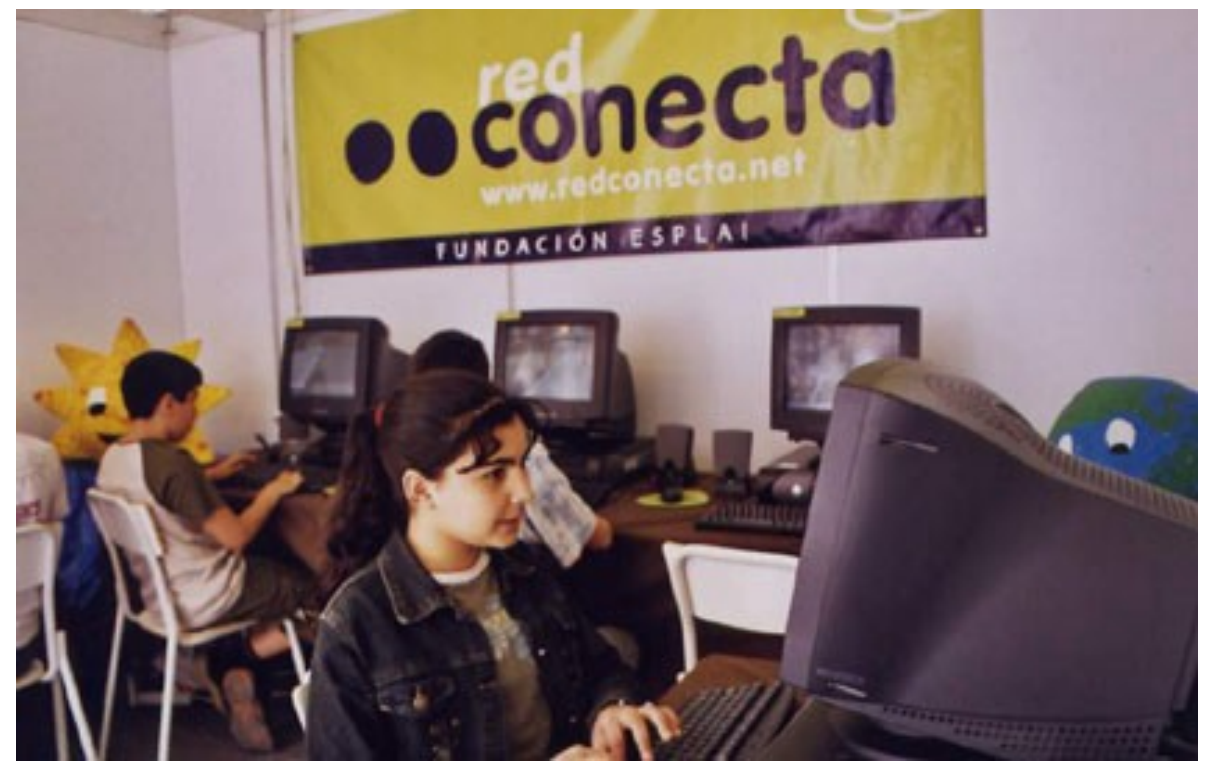

Por ello, Red Conecta fundamenta gran parte de su potencial en el hecho de formar parte de una red que comparte recursos, formación, metodología, coordinación y sistemas de evaluación de resultados.

\subsection{EL EQUIPAMIENTO TECNOLÓGICO}

Cada centro cuenta con un equipamiento básico (un mínimo de 9 PC, 2 impresoras, escáner y webcam y conexión en banda ancha). Y, el conjunto de la red cuenta con una plataforma telemática con herramientas y recursos de gestión de los centros, comunicación, intranet, etc.

\section{Líneas de acción y objetivos.}

Los centros de la Red Conecta tienen tres líneas de acción prioritarias:

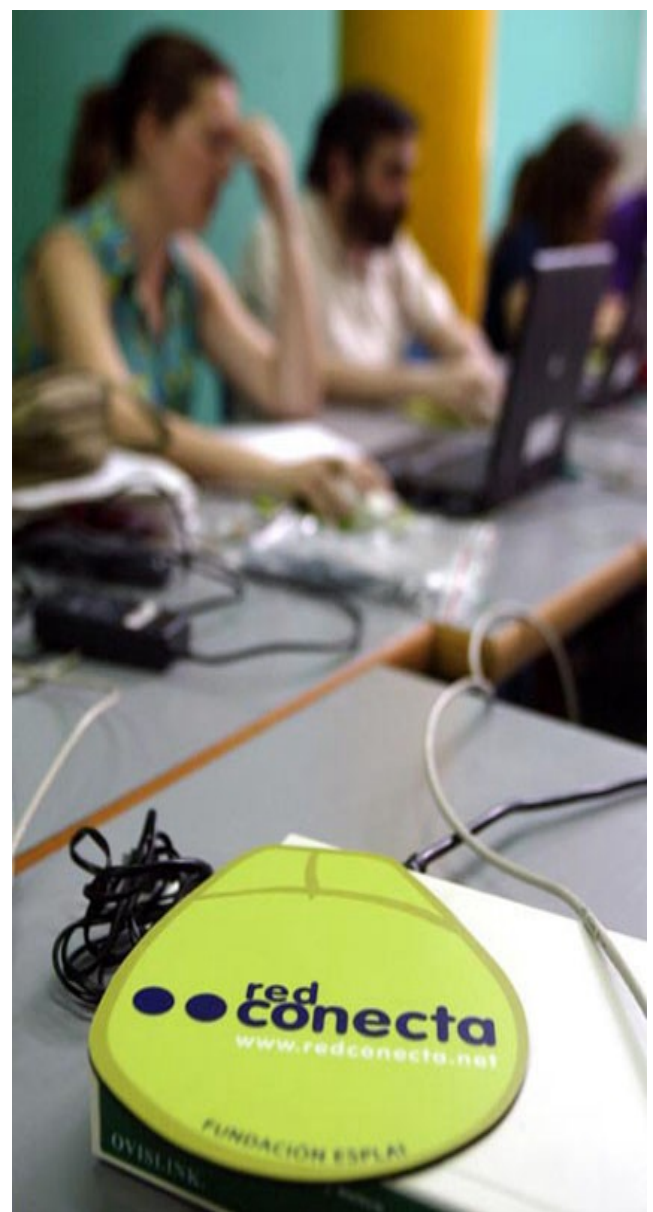

Formación: la formación de personas en el conocimiento y uso de las nuevas tecnologías y a través de ésta incidir en otras áreas de conocimiento, actitudes y habilidades sociales, en definitva la alfabetización digital.

- Uso comunitario: los espacios son accesibles a todo el entorno, especialmente para las entidades y colectivos que trabajan a favor de la inclusión social.

- Inserción laboral: La formación, la mejora de las capacidades y actitudes personales así como la utilización de estrategias de búsqueda activa de empleo serán elementos que entendemos redundan en las posibilidades de inserción en el mercado laboral.

Siendo los objetivos específicos para colectivos y entidades colaboradoras:

- El aumento de la autonomía de las personas en el uso del ordenador, de Internet y de periféricos.

- Una mayor apertura y menor dificultad en el acceso a las nuevas tecnologías por parte de la comunidad en general.

- El refuerzo de la unidad de la comunidad en los barrios a través del uso de los centros como lugar de 
encuentro y como instrumento al servicio del desarrollo del trabajo en red entre asociaciones.

- El aumento del nivel de capacidades, útiles para conseguir un empleo.

\section{Actividades que se realizan}

\subsection{ACCESO PÚBLICO A LOS EQUIPOS Y A INTERNET}

Buena parte del tiempo total de apertura del centro se mantiene como acceso público, en franjas horarias distribuidas a lo largo del día para que se adapten a los diferentes tipos de colectivos: amas de casa, jubilados, estudiantes, empleados tras su jornada laboral, etc.

El dinamizador ofrece soporte a los usuarios en el uso de los equipos y aplicaciones, localización de contenidos, navegación, o la información que se les requiera. Es un espacio muy utilizado para la consulta, trabajos personales, comunicación, búsqueda de empleo, etc.

\subsection{FORMACIÓN}

Actividades orientadas básicamente a la capacitación para un desempeño lo más autónomo posible en el uso del ordenador y aplicaciones básicas de informática. Ello requiere un proceso, que puede variar en su duración según las capacidades y el nivel de partida, guiado por el dinamizador y basado en una aplicación interactiva y manuales desarrollados específicamente.

Las actividades o cursos de formación tienen un horario, una duración determinada y un programa, y los participantes deben inscribirse.

\section{Los cursos de formación}

La actividad básica es la de alfabetización digital complementada por otros módulos con contenidos mas avanzados, en especial Web y multimedia.

Los 7 módulos de alfabetización digital tienen una duración aproximada de 50 horas, tratando los siguientes temas:

- Iniciación al ordenador

- Internet y correo electrónico

- Edición de texto

- Hoja de cálculo básica

Estos módulos formativos se encuentran disponibles en diferentes formatos, manuales en papel, CD interactivo y Web, disponen de un amplio conjunto de fichas, propuestas y ejercicios, así como fichas de auto evaluación que permiten pasar de uno a otro al ritmo de cada persona. Para su uso existe un breve manual para los participantes y una guía para los dinamizadores.

Estos contenidos y su duración además de otros aspectos metodológicos de trabajo colectivo, motivación y seguimiento personalizado contemplados en el programa, son las claves para establecer un proceso de alfabetización digital.

También se realizan cursos más especializados en función de las necesidades y de las demandas de diferentes colectivos y entidades, como formación en herramientas Web y multimedia.

\section{Circuitos de autoformación (fichas, ejercicios)}

Son procesos de autoaprendizaje una vez se dispone de la mínima autonomía, basados en manuales con el apoyo individual del dinamizador. Los materiales están adaptados a los diferentes colectivos.

Esta formación permite profundizar en aspectos concretos o complementar la formación a su propio ritmo. Deben reservar el horario para poder usar el equipo correspondiente.

\subsection{PROYECTOS COLECTIVOS}




\section{Grupos de trabajo / usos comunitarios}

Los centros de la Red Conecta están a disposición de las asociaciones o grupos locales, para proyectos que implican el uso de la informática y comportan un aprendizaje práctico orientado a fines concretos. (La revista del barrio, la organización de una excursión, el fichero de una asociación...). La utilización por parte de otras entidades tiene un efecto de promoción efectivo en el uso del centro.

\section{Grupos de interés}

Se intenta potenciar el uso de Internet desde diferentes perspectivas.

Partiendo de las motivaciones de las personas, se organizan grupos en torno a un centro de interés común. Los temas de trabajo dependen de cada grupo y normalmente suelen ser aficiones, temas cívicos, de solidaridad, de medio ambiente, etc.

\section{Metodología y formación de dinamizadores}

\subsection{DESARROLLO DE METODOLOGÍA PARA COLECTIVOS ESPECÍFICOS}

Estamos desarrollando nuevos recursos de alfabetización digital (fichas, manuales, ejercicios, animaciones...) para colectivos específicos (jóvenes, mayores, personas con poca formación), esto representa una actualización y adaptación constante de las herramientas y la metodología a las necesidades e intereses de las personas participantes.

Para ello se realizan acciones como: producción de recursos en cada centro, puesta en común y estructuración, seguimiento e identificación de buenas prácticas, jornadas presenciales teóricas y/o de intercambios de recursos, tutorías, etc.

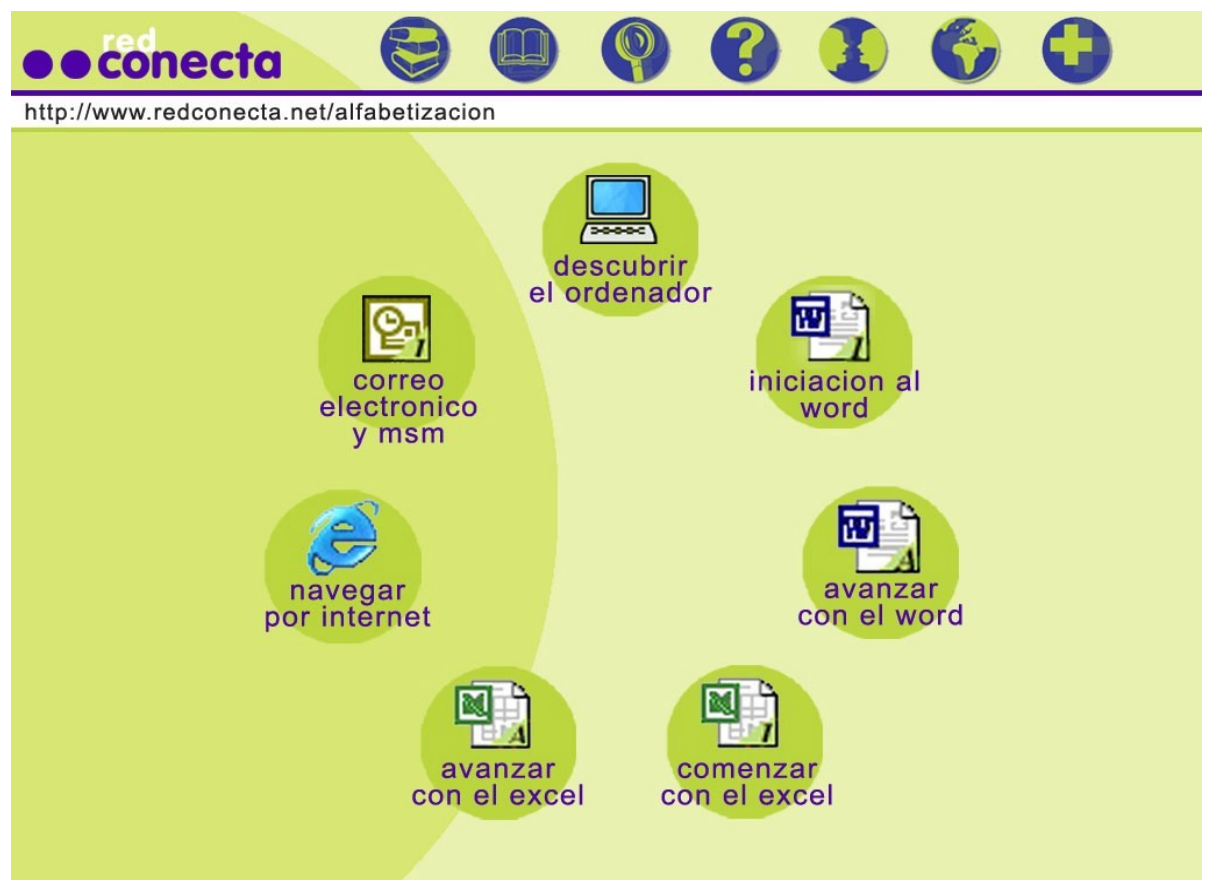

\subsection{FORMACIÓN DE DINAMIZADORES}

Para atender las necesidades de las personas participantes en los centros es requisito indispensable un buen nivel de capacitación de los dinamizadores.

Para ello, la formación es esencial y se realizan anualmente las siguientes actividades:

- 1 curso de formación general de 40 horas (incluye formación tecnológica y de gestión de los centros)

- 1 curso de intervención especializada con diferentes colectivos (jóvenes, mayores, inmigrantes...).

- 1 curso de herramientas Web.

- Jornadas de trabajo e intercambio sobre estrategias de superación de la brecha digital.

\subsection{COORDINACIÓN}


La red cuenta con un equipo de coordinación, apoyo y asistencia que potencia el trabajo en red entre centros, la mejora constante y el aprovechamiento de los recursos colectivos para la resolución de problemas, así como la armonización de estrategias.

Se realiza con regularidad a través de visitas de coordinación a los centros y reuniones de trabajo de seguimiento de objetivos y tutoría.

En las reuniones de coordinación se analiza el funcionamiento de los centros, las actividades de promoción, los resultados de participación y los progresos de los usuarios.

\section{Evaluación de resultados.}

\subsection{RESULTADOS CUANTITATIVOS}

Un aspecto clave en el proceso es la evaluación y para ello se realizan informes periódicos de funcionamiento, aportando resultados sobre cada uno de los centros y del conjunto de la red. Una aplicación informática permite hacer un seguimiento individualizado y la monitorización de la actividad.

Presentamos los siguientes datos generales del funcionamiento de los centros en el periodo comprendido entre noviembre de 2001 y julio de 2004:

\begin{tabular}{|l||l||}
\hline Número de centros implantados & 41 \\
\hline Participantes directos & 12.659 personas en cursos / 7.854 personas en acceso libre. \\
\hline \hline Cursos impartidos & 1.042 cursos realizados. \\
\hline \hline Cursos impartidos & 237.824 \\
\hline $\begin{array}{l}\text { Asociaciones participantes y } \\
\text { vinculadas }\end{array}$ & 78 \\
\hline \hline & 43 Dinamizadores. \\
Equipo Humano & 58 profesionales de apoyo \\
& 72 personas voluntarias. \\
\hline
\end{tabular}

\subsection{EVALUACIÓN EXTERNA DE IMPACTO}

Realizada por el Institut DEP, consultora estratégica, la evaluación externa realizada en 2002 aportó conclusiones muy positivas sobre los logros en cuanto a los beneficios para las personas: mejora de la autoestima, autonomía en el uso del ordenador, confianza en la propia capacidad de aprendizaje, y adquisición de conocimientos.

Entre las claves que contribuyen a estos resultados, el estudio destaca los elementos expuestos en apartados anteriores como el papel del dinamizador, la adaptación de la metodología y recursos, la heterogeneidad de las personas participantes, la adaptación de la propuesta a cada realidad territorial, y la integración de medidas en el contexto de la entidad.

Los objetivos y metodología del estudio de impacto fueron los siguientes[3]:

\section{Objetivos}

\section{A. Evaluar el impacto del programa en los usuarios/as:}

- Determinar el grado de alfabetización digital. 
- Determinar el grado de reducción del riesgo de exclusión social.

- Detección de factores clave en la alfabetización digital y la inclusión social.

B. Evaluar el impacto en la comunidad.

C. Evaluar el impacto en la preparación de los dinamizadores/as.

D. Analizar el servicio ofrecido desde la red.

\section{Metodología}

El estudio de evaluación se organizó en 4 fases de recopilación de información y 1 fase de conclusiones e informe final.

A. Primera fase: cuestionario de dinamizadores estructurado y preparado para ser autoadministrado.

B. Segunda fase: sesión de debate de dinamizadores. 3 grupos de debate conducidos por un profesional. C. Tercera fase: cuestionario de usuarios estructurado, preparado para ser administrado de forma personal por el dinamizador del centro.

D. Cuarta fase: grupos de discusión de usuarios.

E. Quinta fase: análisis de resultados, informe y conclusiones.

\section{Lecciones aprendidas}

\subsection{LAS PERSONAS DINAMIZADORAS, EL ACTIVO PRINCIPAL}

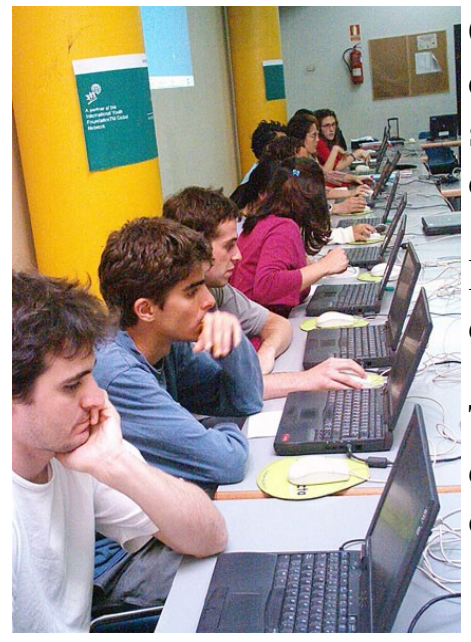

Como hemos comentado la relación con el dinamizador es un factor decisivo en la satisfacción de los participantes. Es la persona de referencia, que con su actitud, su implicación personal y su acción va a motivar y a dar confianza, contribuyendo a que sean protagonistas de sus propios progresos.

Los dinamizadores participan en un proceso de formación y de una corriente de creación colectiva de conocimiento.

Todo ello repercute en la actividad con las personas participantes en el estilo de relación, las estrategias de motivación, la organización de las actividades, o los instrumentos de apoyo al aprendizaje.

\subsection{TRABAJAR EN RED: APORTANDO CADA UNO, GANAMOS TODOS}

La coordinación entre todos los centros implica el compromiso de cada una de las partes para avanzar coordinadamente, mancomunar los esfuerzos para buscar recursos, evaluar los resultados con una misma sistemática y construir un discurso común, basado en la práctica, que pueda incidir de forma real en la sociedad.

Y formar parte de una red reporta efectos positivos: se incrementa el impacto de la actividad al llegar a un mayor y más variado número de personas; se mejora la eficiencia pues la coordinación y determinados servicios compartidos (formación, plataforma, recursos de aprendizaje, etc.) se rentabilizan mejor; y se gana en sostenibilidad.

En términos generales, este modo de trabajo se orienta a incrementar el capital intangible de la red, haciendo aflorar el conocimiento implícito que cada actor de la red acumula a partir de la interacción cotidiana con los participantes y el entorno de los centros.

\subsection{APRENDER CON LA INFORMÁTICA: DEL RECURSO AL EJE TRANSVERSAL}

En el mundo escolar ya se empieza a ver claro, hay que pasar de tener un aula llena de ordenadores a 
la que se va a "usar el ordenador", a tener ordenadores (y en algunos casos Tablet PC) en la misma aula donde se trabaja el resto de contenidos. La informática y sus herramientas pasan a ocupar el lugar instrumental que les corresponde. Se aprende y se trabaja "con" la informática, en un proceso de apropiación que permite pensar, ser críticos, poner a la persona como protagonista, y el conocimiento como el bien colectivo, el que aporta valor.

\subsection{INTEGRACIÓN DE MEDIDAS PARA LA INCLUSIÓN SOCIAL}

La integración de medidas y procesos para favorecer la inclusión social de las personas es una de las estrategias compartidas en la Red Conecta. La alfabetización tecnológica no es una acción aislada, sin contexto, sino que forma parte de un conjunto de actividades formativas y procesos de participación social dirigidos a las personas.

En las asociaciones donde se ubican los centros de la red, las personas participantes no sólo aprenden a usar un ordenador, sino que se comunican con otras personas, asumen responsabilidades, se interesan por otros temas, y en definitiva, desarrollan su capacidad de formar parte de un colectivo y de construir su propia identidad.

Todo ello contribuye a su inclusión social. Al mismo tiempo, personas que participan en la entidad por otros temas (orientación laboral, formación básica, formación para los temas, empleo, asistencia personal...), entran en contacto con la informática y se plantean un aprendizaje que en otro contexto habrían descartado.

\subsection{LAS ASOCIACIONES SON AGENTES PRIVILEGIADOS DE INCLUSIÓN SOCIAL}

Como se ha descrito en el modelo, el proyecto se realiza en colaboración con ONG's, asociaciones y fundaciones de acción social de carácter local con posicionamiento y experiencia en el trabajo con colectivos vulnerables, formando parte de una red en torno al proyecto.

Fundamentalmente se basa en el protagonismo del tercer sector para la superación de la brecha digital y potencia el trabajo en red entre asociaciones para aprovechar la sinergia de cada uno de los diferentes programas.

Esta red tiene como resultado un factor multiplicador de los efectos del proyecto en cada entorno, pues además del trabajo directo con los colectivos de personas destinatarias, también contempla el trabajo con otras entidades locales.

Uno de los hitos perseguidos es que las entidades colaboradoras establezcan acuerdos con otras entidades del territorio para su participación en los centros. Actualmente 78 organizaciones se benefician del proyecto.

En definitiva, para las organizaciones locales, disponer de un recurso como el centro significa una mejora de la capacidad de la entidad para dar respuesta directa a problemáticas sociales de su comunidad y una palanca de desarrollo de sus programas. Para ello, las entidades locales deben actuar desde la corresponsabilidad y el máximo nivel de gestión.

\subsection{LA SUMA DE ESFUERZOS DESDE LOS DIFERENTES SECTORES ES CLAVE}

La superación de la brecha digital requiere aunar esfuerzos entre el tejido social, las administraciones y las empresas, implicando a todos los sectores.

Las propuestas que incluyen a todos se encuentran en mejores condiciones para llegar a las personas, con más posibilidades de viabilidad económica y social y pueden ampliar su alcance de forma muy significativa.

Así mismo debemos contar con los instrumentos adecuados de formación y capacitación del equipo 
humano, de recursos metodológicos para la formación de los participantes, de gestión de los centros y de coordinación y seguimiento[4].

\section{Notas}

[1] Ver vídeo en http://www.redconecta.net/ver-video.asp

[2] En España, la población que accede a Internet es del 33,1\%. Por clase social, la clase media-baja accede en un 17,6\% mientras que la clase baja solamente un 1,6\% (datos de los meses entre abrilmayo del Estudio General de Medios, EGM).

[3] Para más información se puede visitar la página http://www.redconecta.net/DEP/Evaluación DEP.pdf

[4] Más información en www.redconecta.net

(C) Ediciones Universidad de Salamanca 